\title{
Solitons and Other Solutions to Perturbed Rosenau-KdV-RLW Equation with Power Law Nonlinearity
}

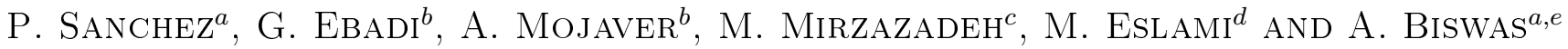

${ }^{a}$ Department of Mathematical Sciences, Delaware State University, Dover, DE 19901-2277, USA

${ }^{b}$ Department of Mathematical Sciences, University of Tabriz, Tabriz, 51666-14766, Iran

${ }^{c}$ Department of Engineering Sciences, Faculty of Technology and Engineering East of Guilan, University of Guilan,

P.C. 44891-63157, Rudsar-Vajargah, Iran

${ }^{d}$ Department of Mathematics, Faculty of Mathematical Sciences, University of Mazandaran, Babolsar, Iran

${ }^{e}$ Department of Mathematics, Faculty of Sciences, King Abdulaziz University, Jeddah-21589, Saudi Arabia (Received January 13, 2015; in final form April 30, 2015)

This paper obtains solitons and other solutions to the perturbed Rosenau-KdV-RLW equation that is used to model dispersive shallow water waves. This equation is taken with power law nonlinearity in this paper. There are several integration tools that are adopted to solve this equation. These are Kudryashov method, sine-cosine function method, $G^{\prime} / G$-expansion scheme and finally the exp-function approach. Solitons and other solutions are obtained along with several constraint conditions that naturally emerge from the structure of these solutions.

DOI: 10.12693/APhysPolA.127.1577

PACS: 05.45.Yv, 02.30.Jr

\section{Introduction}

There are several models describing the dynamics of shallow water waves that appear along lake shores and beaches [1-30]. These are Korteweg-de Vries (KdV) equation [1, 21], the Boussinesq equation, the Kawahara equation, the Peregrine equation or the Benjamin-BonaMahoney equation that is also known as regularized longwave (RLW) equation and several others. However, there is a growing interest in addressing models that describe the dynamics of dispersive shallow water waves. A couple of these models are the Rosenau-Kawahara equation, the Rosenau-KdV ( $\mathrm{R}-\mathrm{KdV})$ equation as well as the Rosenau-KdV-RLW (R-KdV-RLW) equation. This paper describes the dynamics of shallow water waves with $\mathrm{R}-\mathrm{KdV}-\mathrm{RLW}$ equation. In order to keep it on a generalized setting, this model is studied with power law nonlinearity so that results of all special cases are immediate from the ones reported in this paper.

Integrability aspects, conservation laws and perturbation theory are of general interest for such models. This paper addresses the issue of integrability for $\mathrm{R}-\mathrm{KdV}-$ RLW equation with power law nonlinearity in presence of perturbation terms. However, results for conservation laws and soliton perturbation theory are all reported earlier [16-19]. There are several algorithms for integration that will be adopted in this paper. These are Kudryashov method, sine-cosine function approach, $G^{\prime} / G$-expansion

scheme, and finally the exp-function method. Solitary wave solutions and singular periodic solutions are obtained in this paper. These are described in detail in the next few sections. The results of these methods appear with necessary constraint conditions that are listed corresponding to the solutions.

\section{Governing equation}

The perturbed R-KdV-RLW equation under study is given by $[17-19]$ :

$$
u_{t}+a u_{x}+b_{1} u_{x x x}+b_{2} u_{x x t}+c u_{x x x x t}+k\left(u^{n}\right)_{x}=R,
$$

where $R$ represents the perturbation terms which are given by

$$
\begin{aligned}
& R=\alpha u+\beta u_{x x}+\gamma u_{x} u_{x x}+\delta u^{m} u_{x}+\lambda u u_{x x x} \\
& \quad+\nu u u_{x} u_{x x}+\sigma u_{x}^{3}+\xi u_{x} u_{x x x x}+\eta u_{x x} u_{x x x} \\
& \quad+\rho u_{x x x x}+\psi u_{x x x x x}+\kappa u u_{x x x x x} .
\end{aligned}
$$

From (2), the shoaling effect is captured by $\alpha$, while $\beta$ introduces dissipation. Higher-order nonlinear dispersion is indicated by the coefficient of $\delta$. Fifth-order spatial dispersion is given by the coefficient of $\psi$, while higherorder stabilization is introduced by $\rho$. The rest of the terms are accounted for by the Whitham hierarchy.

The exact 1-soliton solution of Eq. (1) is given by $[17-19]$ :

$$
u(x, t)=A \operatorname{sech}^{\frac{4}{n-1}}[B(x-v t)],
$$

where the amplitude $A$ is given by

$$
A=\left\{\frac{\left[-(a c+\psi)\left(n^{2}+2 n+5\right)+D_{1}\right]^{2}(n+3)(3 n+1)}{\left\{8 b_{2}\left(c b_{1}+b_{2} \psi\right)(n+1)^{2}+c\left[-(a c+\psi)\left(n^{2}+2 n+5\right)+D_{1}\right]\left(n^{2}+2 n+5\right)\right\} 16 k(n+1)}\right\}^{\frac{1}{n-1}}
$$

and the inverse width $B$ is

$$
B=\frac{n-1}{n+1}\left[\frac{-(a c+\psi)\left(n^{2}+2 n+5\right)+D_{1}}{32\left(c b_{1}+b_{2} \psi\right)}\right]^{\frac{1}{2}}
$$

corresponding author; e-mail: biswas.anjan@gmail.com with

$$
\begin{aligned}
& D_{1}=\left[(a c+\psi)^{2}\left(n^{2}+2 n+5\right)^{2}\right. \\
& \left.\quad+16\left(c b_{1}+b_{2} \psi\right)\left(b_{1}-a b_{2}\right)(n+1)^{2}\right]^{\frac{1}{2}} .
\end{aligned}
$$

The speed of the dispersive solitary wave is

$$
v=\frac{a(n-1)^{4}+16 b_{1} B^{2}(n-1)^{2}-256 \psi B^{4}}{(n-1)^{4}+16 b_{2} B^{2}(n-1)^{2}+256 c B^{4}},
$$




$$
v=\frac{b_{1}(n-1)^{2}-4 \psi B^{2}\left(n^{2}+2 n+5\right)}{b_{2}(n-1)^{2}+4 c B^{2}\left(n^{2}+2 n+5\right)} .
$$

The appropriate constraints are

$$
\left(b_{1} c+b_{2} \psi\right)\left\{D_{1}-\left(n^{2}+2 n+5\right)(a c+\psi)\right\}>0
$$

and

$$
\begin{aligned}
& (a c+\psi)^{2}\left(n^{2}+2 n+5\right)^{2} \\
& \quad+16(n+1)^{2}\left(b_{1} c+b_{2} \psi\right)\left(b_{1}-a b_{2}\right)>0 .
\end{aligned}
$$

for the solitary waves to exist.

\section{Kudryashov method}

The modification of truncated expansion method, which is a direct and effective algebraic method for computing exact traveling wave solutions, was first proposed by Kudryashov [4]. The modification of truncated expansion method that is known as the Kudryashov method is one of the most effective methods for finding the exact solution of high order NLPDEs [4]. The most complete description of this method was given in [5]. The successful application of this method to NLPDEs was performed in works [6-9].

\subsection{Brief description of the method}

Let us present the algorithm of modification of truncated expansion method (the Kudryashov method) for finding exact solutions of nonlinear PDEs. We consider the nonlinear PDE in the following form:

$$
P_{1}\left(u, u_{t}, u_{x}, u_{x x}, \ldots\right)=0 .
$$

Using traveling wave $u(x, t)=U(z), z=x-v t$ carries Eq. (11) into the following ordinary differential equation (ODE):

$$
P_{2}\left(U,-v U_{z}, U_{z}, U_{z z}, \ldots\right)=0 .
$$

The Kudryashov method contains the following steps.

Step-1: We look for exact solution of Eq. (12) in the form

$$
U=\sum_{l=0}^{N} c_{l}(G(z))^{l}
$$

where $c_{l}(l=0,1, \ldots, N)$ are constants to be determined later, such that $c_{N} \neq 0$, while $G(z)$ has the form

$$
G(z)=\frac{1}{1+K \exp (z)}
$$

a solution to the Riccati equation

$$
G^{\prime}(z)=G^{2}(z)-G(z),
$$

where $K$ is an arbitrary constant.

Step-2: We determine the positive integer $N$ in Eq. (13) by considering the homogeneous balance between the highest order derivatives and the nonlinear terms in Eq. (12).

Step-3: We substitute Eq. (13) into Eq. (12), and calculate all the necessary derivatives $U_{z}, U_{z z}, \ldots$ of the unknown function $U(z)$ as follows:

$$
\begin{aligned}
U_{z} & =\sum_{l=1}^{N} c_{l} l(G-1) G^{l}, \\
U_{z z} & =\sum_{l=1}^{N} c_{l} l\left\{(1+l) G^{2}-(2 l+1) G+l\right\} G^{l},
\end{aligned}
$$

and so on. Substituting Eqs. (13), (16) and (17) into Eq. (12), we obtain the polynomial

$$
E_{2}[G(z)]=0 .
$$

Step-4: Collecting all the terms of the same powers of the function $G(z)$ in the polynomial (18) and equating them to zero, we obtain a system of algebraic equations which can be solved by computer programs such as Maple and Mathematica to get the unknown parameters $c_{l}$ and $v$. Consequently, we obtain the exact solutions of Eq. (11).

\subsection{Application to $R-K d V-R L W$ equation}

In this subsection, we will apply the Kudryashov method to obtain the exact solution of the perturbed $\mathrm{R}-\mathrm{KdV}-\mathrm{RLW}$ equation

$$
\begin{aligned}
& u_{t}+a u_{x}+b_{1} u_{x x x}+b_{2} u_{x x t}+c u_{x x x x t}+k\left(u^{n}\right)_{x}= \\
& \quad \psi u_{x x x x x} .
\end{aligned}
$$

First we introduce the wave variable $z=B(x-v t)$ so that $u(x, t)=U(z)$ into Eq. (19) to carry out the model (19) into an ODE given by

$$
\begin{aligned}
& (a-v) U_{z}+B^{2}\left(b_{1}-v b_{2}\right) U_{z z z}-B^{4}(c v+\psi) U_{z z z z z} \\
& \quad+k\left(U^{n}\right)_{z}=0 .
\end{aligned}
$$

Integrating Eq. (20) once with respect to $z$ and taking integration constant to zero yields

$$
\begin{aligned}
& (a-v) U+B^{2}\left(b_{1}-v b_{2}\right) U_{z z} \\
& \quad-B^{4}(c v+\psi) U_{z z z z}+k U^{n}=0 .
\end{aligned}
$$

Balancing $U_{z z z z}$ with $U^{n}$ in Eq. (21) gives

$$
N+4=n N \Leftrightarrow N=\frac{4}{n-1} .
$$

To obtain an analytic solution, $N$ should be an integer. This requires the use of the transformation

$$
U(z)=[V(z)]^{\frac{2}{n-1}} .
$$

that transforms Eq. (21) to

$$
\begin{aligned}
+ & (a-v)(n-1)^{4} V^{4}+B^{2}\left(b_{1}-v b_{2}\right)\left\{2(3-n)(n-1)^{2}\right. \\
& \left.\times V^{2}\left(V_{z}\right)^{2}+2(n-1)^{3} V^{3} V_{z z}\right\}-B^{4}(c v+\psi) \\
& \times\left\{4(2-n)(3-n)(5-3 n)\left(V_{z}\right)^{4}+24(3-n)\right. \\
& \times(2-n)(n-1) V\left(V_{z}\right)^{2} V_{z z}+6(3-n)(n-1)^{2} \\
& \times V^{2}\left(V_{z z}\right)^{2}+8(3-n)(n-1)^{2} V^{2} V_{z} V_{z z z} \\
& \left.+2(n-1)^{3} V^{3} V_{z z z z}\right\}+k(n-1)^{4} V^{6}=0 .
\end{aligned}
$$

Balancing $V^{3} V_{z z z z}$ with $V^{6}$ gives

$$
3 N+N+4=6 N \Leftrightarrow N=2 .
$$

So, the Kudryashov method suggests the use of the finite expansion

$$
V=c_{0}+c_{1} G+c_{2} G^{2} .
$$

Thus, we have derivatives of function $V$ in the following form:

$$
\begin{aligned}
& V_{z}=2 c_{2} G^{3}+\left(c_{1}-2 c_{2}\right) G^{2}-c_{1} G \\
& V_{z z}=6 c_{2} G^{4}+\left(-10 c_{2}+2 c_{1}\right) G^{3} \\
& \quad+\left(-3 c_{1}+4 c_{2}\right) G^{2}+c_{1} G \\
& V_{z z z}=24 c_{2} G^{5}+\left(-54 c_{2}+6 c_{1}\right) G^{4}+\left(-12 c_{1}+38 c_{2}\right) G^{3} \\
& \quad+\left(-8 c_{2}+7 c_{1}\right) G^{2}-c_{1} G
\end{aligned}
$$

and 


$$
\begin{aligned}
& V_{z z z z}=120 c_{2} G^{6}+\left(-336 c_{2}+24 c_{1}\right) G^{5} \\
& \quad+\left(330 c_{2}-60 c_{1}\right) G^{4}+\left(-130 c_{2}+50 c_{1}\right) G^{3} \\
& \quad+\left(-15 c_{1}+16 c_{2}\right) G^{2}+c_{1} G .
\end{aligned}
$$

Substituting Eqs. (27)-(30) into Eq. (24), and equating all the coefficients of powers of $G(z)$ to be zero, we obtain a system of nonlinear algebraic equations and by solving it, we have

$$
\begin{aligned}
v & =\frac{-16 \psi B^{4}+4(n-1)^{2} b_{1} B^{2}+a(n-1)^{4}}{16 c B^{4}+4(n-1)^{2} b_{2} B^{2}+(n-1)^{4}}, \\
v & =\frac{b_{1}(n-1)^{2}-\left(n^{2}+2 n+5\right) \psi B^{2}}{b_{2}(n-1)^{2}+\left(n^{2}+2 n+5\right) c B^{2}}, \\
B & =\frac{n-1}{n+1}\left[\frac{D_{2}-\left(n^{2}+2 n+5\right)(a c+\psi)}{8\left(c b_{1}+\psi b_{2}\right)}\right]^{\frac{1}{2}}, \\
D_{2} & =\left[\left(n^{2}+2 n+5\right)^{2}(a c+\psi)^{2}\right. \\
& \left.-16(n+1)^{2}\left(c b_{1}+\psi b_{2}\right)\left(a b_{2}-b_{1}\right)\right]^{\frac{1}{2}}, \\
c_{1} & =-c_{2}= \pm\left[\frac{8(n+1)(n+3)(3 n+1)(c v+\psi) B^{4}}{k(n-1)^{4}},\right. \\
c_{0} & =0,
\end{aligned}
$$

where $v$ and $B$ could be compared to Eqs. (7), (8), and (5), respectively. Substituting Eq. (31) into Eq. (21) and inserting the result into the transformation (23), we get the exact solution of Eq. (19) as follows:

$$
u(x, t)=\left\{c_{1} K \frac{\mathrm{e}^{B(x-v t)}}{\left(1+K \mathrm{e}^{B(x-v t)}\right)^{2}}\right\}^{\frac{2}{n-1}} .
$$

When $K= \pm 1$, we have the following 1-soliton solution:

$$
u_{1}(x, t)=\left\{ \pm \frac{c_{1}}{4} \operatorname{sech}^{2}\left[\frac{B}{2}(x-v t)\right]\right\}^{\frac{2}{n-1}}
$$

where

$$
\begin{gathered}
\left(\frac{c_{1}}{4}\right)^{\frac{2}{n-1}}= \\
{\left[ \pm(n+3)(3 n+1)\left[D_{2}-\left(n^{2}+2 n+5\right)(a c+\psi)\right]^{2}\right.} \\
/\left(1 6 ( n + 1 ) k \left\{8 b_{2}(n+1)^{2}\left(c b_{1}+\psi b_{2}\right)+c\left(n^{2}+2 n+5\right)\right.\right. \\
\left.\left.\left.\times\left[D_{2}-\left(n^{2}+2 n+5\right)(a c+\psi)\right]\right\}\right)\right]^{\frac{1}{n-1}},
\end{gathered}
$$

which could be compared to the expression of the amplitude $A$ given by Eq. (4).

Remark: The exact solution (32)-(33) is valid only if

$$
\left(c b_{1}+\psi b_{2}\right)\left\{D_{2}-\left(n^{2}+2 n+5\right)(a c+\psi)\right\}>0,
$$

and

$$
\begin{aligned}
& \left(n^{2}+2 n+5\right)^{2}(a c+\psi)^{2} \geq \\
& 16(n+1)^{2}\left(c b_{1}+\psi b_{2}\right)\left(a b_{2}-b_{1}\right) .
\end{aligned}
$$

\subsubsection{Case $I(n=3)$}

For $n=3$ Eq. (21) reduces to

$$
\begin{aligned}
& (a-v) U+B^{2}\left(b_{1}-v b_{2}\right) U_{z z} \\
& \quad-B^{4}(c v+\psi) U_{z z z z}+k U^{3}=0 .
\end{aligned}
$$

Balancing $U_{z z z z}$ with $U^{3}$ in Eq. (35) gives $N=2$.
Therefore, the Kudryashov method introduces the finite expansion

$$
U=c_{0}+c_{1} G+c_{2} G^{2} .
$$

Substituting Eq. (36) into Eq. (35), we obtain the system of algebraic equations in the form

$$
\begin{aligned}
& G^{6}: k c_{2}^{3}-120 c_{2}(c v+\psi) B^{4}=0, \\
& G^{5}: k c_{1} c_{2}^{2}-2\left(4 c_{1}-61 c_{2}\right)(c v+\psi) B^{4}=0, \\
& G^{4}: k c_{2}\left(c_{0} c_{2}+c_{1}^{2}\right)-10\left(11 c_{2}-2 c_{1}\right)(c v+\psi) B^{4} \\
& \quad+2 c_{2}\left(b_{1}-b_{2} v\right) B^{2}=0, \\
& G^{3}: k c_{1}\left(6 c_{0} c_{2}+c_{1}^{2}\right)-10\left(5 c_{1}-13 c_{2}\right)(c v+\psi) B^{4} \\
& \quad+2\left(c_{1}-5 c_{2}\right)\left(b_{1}-b_{2} v\right) B^{2}=0, \\
& G^{2}: 3 k c_{0}\left(c_{0} c_{2}+c_{1}^{2}\right)-\left(16 c_{2}-15 c_{1}\right)(c v+\psi) B^{4} \\
& \quad+\left(4 c_{2}-3 c_{1}\right)\left(b_{1}-b_{2} v\right) B^{2}+c_{2}(a-v)=0, \\
& G^{1}: 3 k c_{0}^{2} c_{1}-c_{1}(c v+\psi) B^{4} \\
& \quad+c_{1}\left(b_{1}-b_{2} v\right) B^{2}+c_{1}(a-v)=0, \\
& G^{0}:(a-v) c_{0}+k c_{0}^{3}=0 .
\end{aligned}
$$

Solving these under-determined algebraic equations, we get the following results:

$$
\begin{aligned}
v & =\frac{a+b_{1} B^{2}-\psi B^{4}}{1+b_{2} B^{2}+c B^{4}}=\frac{a+7 b_{1} B^{2}-31 \psi B^{4}}{1+7 b_{2} B^{2}+31 c B^{4}}, \\
B & =\left\{-5(a c+\psi)+\left[25(a c+\psi)^{2}\right.\right. \\
& \left.\left.-16\left(b_{1} c+b_{2} \psi\right)\left(a b_{2}-b_{1}\right)\right]^{\frac{1}{2}} / 8\left(b_{1} c+b_{2} \psi\right)\right\}^{\frac{1}{2}}, \\
c_{1} & =-c_{2}=\mp\left[\frac{120(c v+\psi) B^{4}}{k}\right]^{\frac{1}{2}}= \\
& \mp\left\{\frac{2 B^{2}\left[65(c v+\psi) B^{2}-\left(b_{1}-b_{2} v\right)\right]}{k}\right\}^{\frac{1}{2}}, \\
c_{0} & =0 .
\end{aligned}
$$

Substituting Eq. (44) into Eq. (36), we get the exact solution of Eq. (19) as follows:

$$
u(x, t)=c_{1} \frac{K \mathrm{e}^{B(x-v t)}}{\left(1+K \mathrm{e}^{B(x-v t)}\right)^{2}} .
$$

When $K=1$, we have the following solitary wave solution:

$$
u_{3}(x, t)=\frac{c_{1}}{4} \operatorname{sech}^{2}\left[\frac{B}{2}(x-v t)\right] .
$$

When $K=-1$, the following singular 1-soliton solution is obtained:

$$
u_{4}(x, t)=-\frac{c_{1}}{4} \operatorname{csch}^{2}\left[\frac{B}{2}(x-v t)\right] .
$$

Remark: The exact solutions (45)-(47) are valid only if

$$
\begin{aligned}
& \left\{\left[25(a c+\psi)^{2}-16\left(b_{1} c+b_{2} \psi\right)\left(a b_{2}-b_{1}\right)\right]^{\frac{1}{2}}\right. \\
& \quad-5(a c+\psi)\}\left(b_{1} c+b_{2} \psi\right)>0,
\end{aligned}
$$

where

$$
25(a c+\psi)^{2} \geq 16\left(b_{1} c+b_{2} \psi\right)\left(a b_{2}-b_{1}\right)
$$

and

$$
\left[65(c v+\psi) B^{2}-\left(b_{1}-b_{2} v\right)\right] k>0 .
$$




\subsubsection{Case II $(n=5)$}

For $n=5$, Eq. (21) reduces to

$$
\begin{aligned}
& (a-v) U+B^{2}\left(b_{1}-v b_{2}\right) U_{z z}-B^{4}(c v+\psi) U_{z z z z} \\
& \quad+k U^{5}=0 .
\end{aligned}
$$

Balancing $U_{z z z z}$ with $U^{5}$ in Eq. (48) gives $N=1$. Thus, the Kudryashov method suggests the use of the finite expansion

$$
U=c_{0}+c_{1} G .
$$

Substituting Eq. (49) into Eq. (48) and equating all the coefficients of powers of $G(z)$ to be zero, we obtain

$$
\begin{aligned}
& G^{5}: k c_{1}^{5}-24 c_{1}(c v+\psi) B^{4}=0, \\
& G^{4}: 5 k c_{0} c_{1}^{4}+60 c_{1}(c v+\psi) B^{4}=0, \\
& G^{3}: 10 k c_{0}^{2} c_{1}^{3}-50 c_{1}(c v+\psi) B^{4} \\
& \quad+2 c_{1}\left(b_{1}-v b_{2}\right) B^{2}=0, \\
& G^{2}: 10 k c_{0}^{3} c_{1}^{2}+15 c_{1}(c v+\psi) B^{4} \\
& \quad-3 c_{1}\left(b_{1}-v b_{2}\right) B^{2}=0, \\
& G^{1}:(a-v) c_{1}+5 k c_{0}^{4} c_{1}-c_{1}(c v+\psi) B^{4} \\
& \quad+c_{1}\left(b_{1}-v b_{2}\right) B^{2}=0, \\
& G^{0}:(a-v) c_{0}+k c_{0}^{5}=0 .
\end{aligned}
$$

With the aid of Maple, we find the special solution of the above system as

$$
\begin{aligned}
v & =\frac{4 a-b_{1} B^{2}+\psi B^{4}}{4-b_{2} B^{2}-c B^{4}} \\
B & =\left(\left\{10(a c+\psi)+\left[100(a c+\psi)^{2}\right.\right.\right. \\
& \left.\left.\left.-24\left(a b_{2}-b_{1}\right)\left(b_{1} c+b_{2} \psi\right)\right]^{\frac{1}{2}}\right\} / 6\left(b_{1} c+b_{2} \psi\right)\right)^{\frac{1}{2}}, \\
c_{1} & =-2 c_{0}= \pm 2\left[\frac{3(c v+\psi) B^{4}}{2 k}\right]^{\frac{1}{4}}= \pm 2\left[\frac{v-a}{k}\right]^{\frac{1}{4}}
\end{aligned}
$$

Substituting Eq. (56) into Eq. (49), we recover the exact solutions of Eq. (19) as follows:

$$
\begin{aligned}
& u_{5}(x, t)=c_{0} \tanh [B(x-v t)], \\
& u_{6}(x, t)=c_{0} \operatorname{coth}[B(x-v t)] .
\end{aligned}
$$

These are shock wave solution and singular solitary wave solution respectively.

Remark: The exact solutions (57)-(58) are valid only if

$$
\begin{aligned}
& \left\{\left[100(a c+\psi)^{2}-24\left(a b_{2}-b_{1}\right)\left(b_{1} c+b_{2} \psi\right)\right]^{\frac{1}{2}}\right. \\
& \quad+10(a c+\psi)\}\left(b_{1} c+b_{2} \psi\right)>0
\end{aligned}
$$

where

$$
100(a c+\psi)^{2} \geq 24\left(a b_{2}-b_{1}\right)\left(b_{1} c+b_{2} \psi\right)
$$

and

$$
(v-a) k>0, \quad(c v+\psi) k>0
$$

Remark: Razborova et al. [2] showed that for the perturbed R-KdV-RLW equation, the topological solitons would exist only for $n=3,5$ and no other value of $n$ can be permitted for the topological soliton solutions to be valid.

\section{Sine-cosine function method}

This integration technique is another mathematical mechanism to extract soliton and other solutions to any NLPDE [10, 11].

\subsection{Overview of the method}

\section{A PDE}

$$
P\left(u, u_{t}, u_{x}, u_{x x}, u_{x t}, u_{t t}, \ldots\right)=0,
$$

can be converted to an ODE

$$
Q\left(U, U^{\prime}, U^{\prime \prime}, \ldots\right)=0,
$$

upon using a traveling wave variable $u(x, t)=U(z), z=$ $x$-vt. If possible, integrate Eq. (60) term by term one or more times. This will reduce the order of Eq. (60). For simplicity, the integration constants can be set to zero. The solutions of the reduced ODE can be expressed in the form

$$
U(z)=\lambda \cos ^{\beta}(\mu z), \quad|z| \leq \frac{\pi}{2 \mu},
$$

or in the form

$$
U(z)=\lambda \sin ^{\beta}(\mu z), \quad|z| \leq \frac{\pi}{\mu},
$$

where $\lambda, \mu$, and $\beta$ are parameters that will be determined, $\mu$ and $v$ are the wave number and the wave speed respectively. These assumptions give

$$
\begin{aligned}
& \left(U^{n}\right)^{\prime \prime}=-n^{2} \mu^{2} \beta^{2} \lambda^{n} \cos ^{n \beta}(\mu z) \\
& \quad+n \mu^{2} \lambda^{n} \beta(n \beta-1) \cos ^{n \beta-2}(\mu z),
\end{aligned}
$$

and

$$
\begin{aligned}
& \left(U^{n}\right)^{\prime \prime}=-n^{2} \mu^{2} \beta^{2} \lambda^{n} \sin ^{n \beta}(\mu z) \\
& +n \mu^{2} \lambda^{n} \beta(n \beta-1) \sin ^{n \beta-2}(\mu z) .
\end{aligned}
$$

Using Eqs. (61)-(64) in the reduced ODE gives a trigonometric equation in $\cos ^{K}(z)$ or $\sin ^{K}(z)$ terms. The parameters are then determined by first balancing the exponents of each pair of cosines or sines to determine $K$. We next collect all coefficients of the same power in $\cos ^{K}(z)$ or $\sin ^{K}(z)$, where these coefficients have to vanish. This gives a system of algebraic equations among the unknowns $\beta, \lambda, v$ and $\mu$ that will be determined. The solutions proposed in Eqs. (61) and (62) follow immediately.

\subsection{Application to $R-K d V-R L W$ equation}

We will use the sine-cosine function method to handle the perturbed $\mathrm{R}-\mathrm{KdV}-\mathrm{RLW}$ equation. Using the wave transformation

$$
u(x, t)=U(z), \quad z=x-v t,
$$

and integrating the resultant equation once with respect to $z$ and taking integration constant to zero, we have

$$
\begin{aligned}
& (a-v) U+\left(b_{1}-v b_{2}\right) U_{z z}-(c v+\psi) U_{z z z z} \\
& \quad+k U^{n}=0 .
\end{aligned}
$$

Using the assumption

$$
U(z)=\lambda \cos ^{\beta}(\mu z),
$$

in Eq. (66) we obtain

$$
\begin{aligned}
& U_{z z}=-\mu^{2} \beta^{2} \lambda \cos ^{\beta}(\mu z)+\mu^{2} \lambda \beta(\beta-1) \cos ^{\beta-2}(\mu z), \\
& U_{z z z z}=\mu^{4} \beta^{4} \lambda \cos ^{\beta}(\mu z) \\
& \quad-2 \mu^{4} \lambda \beta(\beta-1)\left(\beta^{2}-2 \beta+2\right) \cos ^{\beta-2}(\mu z)
\end{aligned}
$$




$$
+\mu^{4} \lambda \beta(\beta-1)(\beta-2)(\beta-3) \cos ^{\beta-4}(\mu z) .
$$

Substituting Eqs. (67)-(69) into Eq. (66), we have

$$
\begin{aligned}
\lambda\{(a & \left.-v)-\left(b_{1}-v b_{2}\right) \mu^{2} \beta^{2}-(c v+\psi) \mu^{4} \beta^{4}\right\} \cos ^{\beta}(\mu z) \\
& +\lambda\left\{\left(b_{1}-v b_{2}\right) \mu^{2} \beta(\beta-1)\right. \\
& \left.+2(c v+\psi) \mu^{4} \beta(\beta-1)\left(\beta^{2}-2 \beta+2\right)\right\} \cos ^{\beta-2}(\mu z) \\
& -\lambda(c v+\psi) \mu^{4} \beta(\beta-1)(\beta-2)(\beta-3) \cos ^{\beta-4}(\mu z) \\
& +k \lambda^{n} \cos ^{n \beta}(\mu z)=0 .
\end{aligned}
$$

Using the balance method, by equating the exponents and the coefficients of $\cos ^{K}$, we get

$$
\begin{aligned}
& \beta(\beta-1)(\beta-2)(\beta-3) \neq 0 \\
& n \beta=\beta-4, \\
& k \lambda^{n}-\lambda(c v+\psi) \mu^{4} \beta(\beta-1)(\beta-2)(\beta-3)=0, \\
& (a-v)-\left(b_{1}-v b_{2}\right) \mu^{2} \beta^{2}-(c v+\psi) \mu^{4} \beta^{4}=0, \\
& \left(b_{1}-v b_{2}\right)+2(c v+\psi) \mu^{2}\left(\beta^{2}-2 \beta+2\right)=0 .
\end{aligned}
$$

Solving the system (Eqs. (71)-(75)) simultaneously, we get the solutions set

$$
\begin{gathered}
\lambda=\left[\frac{8(c v+\psi)(n+1)(n+3)(3 n+1) \mu^{4}}{k(n-1)^{4}}\right]^{\frac{1}{n-1}}, \\
v=\frac{b_{1}(n-1)^{2}+4 \psi\left(n^{2}+2 n+5\right) \mu^{2}}{b_{2}(n-1)^{2}-4 c\left(n^{2}+2 n+5\right) \mu^{2}}= \\
\frac{a(n-1)^{4}-16 b_{1}(n-1)^{2} \mu^{2}-256 \psi \mu^{4}}{(n-1)^{4}-16 b_{2}(n-1)^{2} \mu^{2}+256 c \mu^{4}}, \\
\mu=\frac{n-1}{n+1}\left[\frac{D_{3}+\left(n^{2}+2 n+5\right)(a c+\psi)}{32\left(c b_{1}+\psi b_{2}\right)}\right]^{\frac{1}{2}}, \\
D_{3}=\left[\left(n^{2}+2 n+5\right)^{2}(a c+\psi)^{2}\right. \\
\left.-16(n+1)^{2}\left(c b_{1}+\psi b_{2}\right)\left(a b_{2}-b_{1}\right)\right]^{\frac{1}{2}}, \\
\beta=-\frac{4}{n-1} .
\end{gathered}
$$

Consequently, we obtain the following singular periodic solutions:

$$
u_{7}(x, t)=\lambda \sec ^{\frac{4}{n-1}}[\mu(x-v t)], \quad|x-v t| \leq \frac{\pi}{2 \mu}
$$

and

$$
u_{8}(x, t)=\lambda \csc ^{\frac{4}{n-1}}[\mu(x-v t)], \quad|x-v t| \leq \frac{\pi}{\mu} .
$$

Remark: The exact solutions (77)-(78) are valid only if

$$
\begin{array}{r}
\left\{\left(n^{2}+2 n+5\right)(a c+\psi)+\left[\left(n^{2}+2 n+5\right)^{2}(a c+\psi)^{2}\right.\right. \\
\left.\left.-16(n+1)^{2}\left(c b_{1}+\psi b_{2}\right)\left(a b_{2}-b_{1}\right)\right]^{\frac{1}{2}}\right\}\left(c b_{1}+\psi b_{2}\right)>0
\end{array}
$$

where

$$
\begin{aligned}
& \left(n^{2}+2 n+5\right)^{2}(a c+\psi)^{2} \geq \\
& \quad 16(n+1)^{2}\left(c b_{1}+\psi b_{2}\right)\left(a b_{2}-b_{1}\right)
\end{aligned}
$$

\section{5. $G^{\prime} / G$-expansion method}

Recently, a new method has been proposed by Wang et al. [18], called the $G^{\prime} / G$-expansion method to study traveling wave solutions of nonlinear evolution equations.
This useful method is developed successfully by many authors [19-22] and the reference therein. The $G^{\prime} / G$ expansion method [18] is based on the assumptions that the traveling wave solutions can be expressed by a polynomial in $G^{\prime} / G$ such that $G=G(z)$ satisfies a second order linear ordinary differential equation (ODE).

\subsection{Review of the algorithm}

We now summarize the $G^{\prime} / G$-expansion method, established in 2011 [19], the details of which can be found in [18-22] among many others.

We assume that the given nonlinear partial differential equation (NLPDE) for $u(x, t)$ is in the form

$$
P\left(u, \frac{\partial u}{\partial t}, \frac{\partial u}{\partial x}, \frac{\partial^{2} u}{\partial x^{2}}, \frac{\partial^{2} u}{\partial t \partial x}, \frac{\partial^{2} u}{\partial t^{2}}, \ldots\right)=0,
$$

where $P$ is a polynomial. The essence of the $G^{\prime} / G_{-}$ expansion method can be presented in the following steps:

Step-1: To find the traveling wave solutions of Eq. (79), we introduce the wave variable

$$
u(x, t)=U(z), \quad z=x-v t .
$$

Substituting Eq. (80) into Eq. (79), we obtain the following ODE:

$$
Q\left(U, \frac{d U}{d z}, \frac{d^{2} U}{d z^{2}}, \ldots\right)=0 .
$$

Step-2: Eq. (81) is then integrated as long as all terms contain derivatives where integration constants are considered zero.

Step-3: Introduce the solution $U(z)$ of Eq. (81) in the finite series form

$$
U(z)=\sum_{l=0}^{N} a_{l}\left(\frac{G^{\prime}(z)}{G(z)}\right)^{l}
$$

where $a_{l}$ are real constants with $a_{N} \neq 0$ and $N$ is a positive integer to be determined. The function $G(z)$ is the solution of the auxiliary linear ordinary differential equation

$$
G^{\prime \prime}(z)+\lambda G^{\prime}(z)+\mu G(z)=0,
$$

where $\lambda$ and $\mu$ are real constants to be determined.

Step-4: Determining $N$, can be accomplished by balancing the linear term of highest order derivatives with the highest order nonlinear term in Eq. (81).

Step-5: Substituting the general solution of (83) together with (82) into Eq. (81) yields an algebraic equation involving powers of $G^{\prime} / G$. Equating the coefficients of each power of $G^{\prime} / G$ to zero gives a system of algebraic equations for $a_{l}, \lambda, \mu$ and $v$. Then, we solve the system with the aid of a computer algebra system, such as Maple, to determine these constants. Next, depending on the sign of the discriminant $\Delta=\lambda^{2}-4 \mu$, we obtain solutions of Eq. (81). So, we can obtain exact solutions of the given Eq. (79).

\subsection{Application to $R-K d V-R L W$ equation}

In this subsection, we will apply the $G^{\prime} / G$-expansion method to handle the perturbed R-KdV-RLW equation. 


\subsubsection{Case I $(n=3)$}

For $n=3$, Eq. (66) reduces to

$$
\begin{aligned}
& (a-v) U+\left(b_{1}-v b_{2}\right) U_{z z}-(c v+\psi) U_{z z z z} \\
& \quad+k U^{3}=0 .
\end{aligned}
$$

Balancing $U_{z z z z}$ with $U^{3}$ in Eq. (84) gives $N=2$.

Therefore, the solution of Eq. (84) can be written in the form

$$
\begin{aligned}
U(z) & =a_{0}+a_{1}\left(\frac{G^{\prime}(z)}{G(z)}\right)+a_{2}\left(\frac{G^{\prime}(z)}{G(z)}\right)^{2}, \\
a_{2} & \neq 0,
\end{aligned}
$$

where $G(z)$ satisfies the second-order linear ordinary differential equation

$$
G^{\prime \prime}(z)+\lambda G^{\prime}(z)+\mu G(z)=0,
$$

where $\lambda$ and $\mu$ are real constants to be determined.

From Eqs. (85) and (86) we derive

$$
\begin{aligned}
& U_{z z}=6 a_{2}\left(\frac{G^{\prime}}{G}\right)^{4}+\left(2 a_{1}+10 a_{2} \lambda\right)\left(\frac{G^{\prime}}{G}\right)^{3} \\
& +\left(4 a_{2} \lambda^{2}+8 a_{2} \mu+3 a_{1} \lambda\right)\left(\frac{G^{\prime}}{G}\right)^{2} \\
& \quad+\left(6 a_{2} \lambda \mu+2 a_{1} \mu+a_{1} \lambda^{2}\right)\left(\frac{G^{\prime}}{G}\right)+a_{1} \lambda \mu \\
& \quad+2 a_{2} \mu^{2}, \\
& U_{z} z z=120 a_{2}\left(\frac{G^{\prime}}{G}\right)^{6}+\left(24 a_{1}+366 a_{2} \lambda\right)\left(\frac{G^{\prime}}{G}\right)^{5} \\
& \quad+\left(330 a_{2} \lambda^{2}+240 a_{2} \mu+60 a_{1} \lambda\right)\left(\frac{G^{\prime}}{G}\right)^{4} \\
& \quad+\left(440 a_{2} \lambda \mu+50 a_{1} \lambda^{2}+130 a_{2} \lambda^{3}+40 a_{1} \mu\right)\left(\frac{G^{\prime}}{G}\right)^{3} \\
& +\left(136 a_{2} \mu^{2}+60 a_{1} \lambda \mu+16 a_{2} \lambda^{4}+232 a_{2} \lambda^{2} \mu\right. \\
& \left.\quad+15 a_{1} \lambda^{3}\right)\left(\frac{G^{\prime}}{G}\right)^{2}+\left(a_{1} \lambda^{4}+22 a_{1} \lambda^{2} \mu\right. \\
& \left.+16 a_{1} \mu^{2}+120 a_{2} \mu^{2} \lambda+30 a_{2} \lambda^{3} \mu\right)\left(\frac{G^{\prime}}{G}\right)^{3} \\
& +a_{1} \mu \lambda^{3}+14 a_{2} \lambda^{2} \mu^{2}+8 a_{1} \lambda \mu^{2}+16 a_{2} \mu^{3} .
\end{aligned}
$$

Substituting Eqs. (85)-(88) into Eq. (84), collecting all terms with the same powers of $G^{\prime} / G$ and setting each coefficient to zero, we obtain a system of algebraic equations for $a_{0}, a_{1}, a_{2}, v, \lambda$ and $\mu$ as follows:

$\left(\frac{G^{\prime}}{G}\right)^{6}$ coeff.:

$$
-120(c v+\psi) a_{2}+k a_{2}^{3}=0,
$$

$\left(\frac{G^{\prime}}{G}\right)^{5}$ coeff.:

$$
-(c v+\psi)\left(24 a_{1}+336 a_{2} \lambda\right)+3 k c a_{1} a_{2}^{2}=0,
$$

$\left(\frac{G^{\prime}}{G}\right)^{4}$ coeff.:

$$
\begin{aligned}
& 3 k a_{0} a_{2}^{2}+6\left(b_{1}-v b_{2}\right) a_{2}-(c v+\psi)\left(330 a_{2} \lambda^{2}+240 a_{2} \mu\right. \\
& \left.\quad+60 a_{1} \lambda\right)+3 k a_{1}^{2} a_{2}=0,
\end{aligned}
$$

$\left(\frac{G^{\prime}}{G}\right)^{3}$ coeff.:

$6 k a_{0} a_{1} a_{2}-(c v+\psi)\left(400 a_{2} \lambda \mu+50 a_{1} \lambda^{2}+130 a_{2} \lambda^{3}\right.$

$\left.+40 a_{1} \mu\right)+\left(b_{1}-v b_{2}\right)\left(2 a_{1}+10 a_{2} \lambda\right)+k a_{1}^{3}=0$,

$\left(\frac{G^{\prime}}{G}\right)^{2}$ coeff.:

$$
\begin{gathered}
3 k a_{0}^{2} a_{2}+3 k a_{0} a_{1}^{2}-(c v+\psi)\left(136 a_{2} \mu^{2}+60 a_{1} \lambda \mu\right. \\
\left.\quad+16 a_{2} \lambda^{4}+232 a_{2} \lambda^{2} \mu+15 a_{1} \lambda^{3}\right)+(a-v) a_{2} \\
+\left(b_{1}-v b_{2}\right)\left(4 a_{2} \lambda^{2}+8 a_{2} \mu+3 a_{1} \lambda\right)=0,
\end{gathered}
$$

$\left(\frac{G^{\prime}}{G}\right)^{1}$ coeff.:

$$
\begin{aligned}
& (a-v) a_{1}-(c v+\psi)\left(a_{1} \lambda^{4}+22 a_{1} \lambda^{2} \mu+16 a_{1} \mu^{2}\right. \\
& \left.\quad+120 a_{2} \mu^{2} \lambda+30 a_{2} \lambda^{3} \mu\right) \\
& \quad+\left(b_{1}-v b_{2}\right)\left(6 a_{2} \lambda \mu+2 a_{1} \mu+a_{1} \lambda^{2}\right)+3 k a_{0}^{2} a_{1}=0,
\end{aligned}
$$

$\left(\frac{G^{\prime}}{G}\right)^{0}$ coeff.:

$$
\begin{aligned}
& (a-v) a_{0}+k a_{0}^{3}-(c v+\psi)\left(8 a_{1} \lambda \mu^{2}+16 a_{2} \mu^{3}+a_{1} \mu \lambda^{3}\right. \\
& \left.+14 a_{2} \lambda^{2} \mu^{2}\right)+\left(b_{1}-v b_{2}\right)\left(a_{1} \lambda \mu+2 a_{2} \mu^{2}\right)=0 .
\end{aligned}
$$

With the aid of Maple, we shall find the special solution of the above system

$$
\begin{aligned}
a & =-\frac{1}{3000 k a_{2}^{2} c^{2}}\left(-960 b_{1} c a_{2}^{2} b_{2} k+115200 b_{2} b_{1} c \psi\right. \\
& +57600 \psi^{2} b_{2}^{2}-960 \psi b_{2}^{2} a_{2}^{2} k+57600 b_{1}^{2} c^{2} \\
& \left.+3000 a_{2}^{2} c k \psi+4 a_{2}^{4} b_{2}^{2} k^{2}-25 a_{2}^{4} c k^{2}\right), \\
v & =\frac{k a_{2}^{2}-120 \psi}{120 c} \\
\mu & =\frac{a_{2}^{2} k\left(5 c \lambda^{2}+b_{2}\right)-120\left(b_{1} c+\psi b_{2}\right)}{20 k a_{2}^{2} c} \\
a_{0} & =\frac{a_{2}^{2} k\left(5 c \lambda^{2}+b_{2}\right)-120\left(b_{1} c+\psi b_{2}\right)}{20 k a_{2} c} \\
a_{1} & =a_{2} \lambda,
\end{aligned}
$$

where $a_{2}, \lambda$ are arbitrary constants.

Substituting the solution set (90) into Eq. (85), the solution formula of Eq. (84) can be written as

$$
U(z)=\mu+a_{2} \lambda\left(\frac{G^{\prime}(z)}{G(z)}\right)+a_{2}\left(\frac{G^{\prime}(z)}{G(z)}\right)^{2},
$$

where

$$
\begin{aligned}
a & =-\frac{1}{3000 k a_{2}^{2} c^{2}}\left(-960 b_{1} c a_{2}^{2} b_{2} k+115200 b_{2} b_{1} c \psi\right. \\
& +57600 \psi^{2} b_{2}^{2}-960 \psi b_{2}^{2} a_{2}^{2} k+57600 b_{1}^{2} c^{2} \\
& \left.+3000 a_{2}^{2} c k \psi+4 a_{2}^{4} b_{2}^{2} k^{2}-25 a_{2}^{4} c k^{2}\right) .
\end{aligned}
$$

Substituting the general solutions of second order linear ODE into Eq. (91) gives two types of traveling wave solutions.

When $\Delta=\lambda^{2}-4 \mu>0$, we have traveling wave solution

$$
\left(\frac{G^{\prime}}{G}\right)=-\frac{\lambda}{2}+\Theta \frac{C_{1} \sinh (\Theta z)+C_{2} \cosh (\Theta z)}{C_{1} \cosh (\Theta z)+C_{2} \sinh (\Theta z)},
$$




$$
\begin{gathered}
\Theta=\frac{\sqrt{\lambda^{2}-4 \mu}}{2}, \\
u_{21}(x, t)=-\Sigma^{2}\left\{1-\left[\frac{C_{1} \sinh (\Sigma z)+C_{2} \cosh (\Sigma z)}{C_{1} \cosh (\Sigma z)+C_{2} \sinh (\Sigma z)}\right]^{2}\right\}, \\
\Sigma=\sqrt{\frac{120\left(b_{1} c+\psi b_{2}\right)-b_{2} k a_{2}^{2}}{20 k c a_{2}}}
\end{gathered}
$$

where $C_{1}$ and $C_{2}$ are arbitrary constants and $z=x-$ $\left\{\frac{k a_{2}^{2}-120 \psi}{120 c}\right\} t$.

As a special case, assuming $C_{1} \neq 0$ and $C_{2}=0$ the traveling wave solution of the perturbed $\mathrm{R}-\mathrm{KdV}-\mathrm{RLW}$ equation can be written as

$$
u_{22}(x, t)=\Sigma^{2} \operatorname{sech}^{2}(\Sigma z),
$$

Next, assuming $C_{1}=0$ and $C_{2} \neq 0$, then we obtain

$$
u_{23}(x, t)=\Sigma^{2} \operatorname{csch}^{2}(\Sigma z) .
$$

which are solitary waves and singular solitary waves, respectively.

When $\Delta=\lambda^{2}-4 \mu<0$, the traveling wave solution is

$$
\begin{aligned}
& \left(\frac{G^{\prime}}{G}\right)=-\frac{\lambda}{2}+\Theta \frac{C_{1} \sin (\Theta z)+C_{2} \cos (\Theta z)}{C_{1} \cos (\Theta z)+C_{2} \sin (\Theta z)}, \\
& \Theta=\frac{\sqrt{\lambda^{2}-4 \mu}}{2}, \\
& u_{24}(x, t)=-\Sigma^{2}\left(1-\left\{\frac{C_{1} \sin (\Sigma z)+C_{2} \cos (\Sigma z)}{C_{1} \cos (\Sigma z)+C_{2} \sin (\Sigma z)}\right\}^{2}\right), \\
& \Sigma=\sqrt{\frac{120\left(b_{1} c+\psi b_{2}\right)-b_{2} k a_{2}^{2}}{20 k c a_{2}}},
\end{aligned}
$$

where $C_{1}$ and $C_{2}$ are arbitrary constants and $z=x-$ $\left\{\frac{k a_{2}^{2}-120 \psi}{120 c}\right\} t$.

Also, with the assumption $C_{1} \neq 0$ and $C_{2}=0$, the special cases are

$$
u_{25}(x, t)=\Sigma^{2} \sec ^{2}(\Sigma z),
$$

and when $C_{1}=0, \quad C_{2} \neq 0$ the solution of the perturbed R-KdV-RLW equation will be

$$
u_{26}(x, t)=\Sigma^{2} \csc ^{2}(\Sigma z) \text {. }
$$

and these are singular periodic solutions.

Remark: The exact solutions (93)-(99) are valid only if

$$
\begin{aligned}
a & =-\frac{1}{3000 k a_{2}^{2} c^{2}}\left(-960 b_{1} c a_{2}^{2} b_{2} k+115200 b_{2} b_{1} c \psi\right. \\
& +57600 \psi^{2} b_{2}^{2}-960 \psi b_{2}^{2} a_{2}^{2} k+57600 b_{1}^{2} c^{2}+3000 a_{2}^{2} c k \psi \\
& \left.+4 a_{2}^{4} b_{2}^{2} k^{2}-25 a_{2}^{4} c k^{2}\right) .
\end{aligned}
$$

\subsubsection{Case $I I(n=5)$}

For $n=5$, Eq. (66) reduces to

$$
\begin{aligned}
& (a-v) U+\left(b_{1}-v b_{2}\right) U_{z z}-(c v+\psi) U_{z z z z} \\
& \quad+k U^{5}=0 .
\end{aligned}
$$

Balancing $U_{z z z z}$ with $U^{5}$ in Eq. (100) gives $N=1$.

Therefore, the solution of Eq. (100) can be written in the form

$$
U(z)=a_{0}+a_{1}\left(\frac{G^{\prime}(z)}{G(z)}\right), \quad a_{1} \neq 0,
$$

where $G(z)$ satisfies the second-order linear ordinary differential equation

$$
G^{\prime \prime}(z)+\lambda G^{\prime}(z)+\mu G(z)=0,
$$

where $\lambda$ and $\mu$ are real constants to be determined.

Substituting Eq. (101) into Eq. (100), collecting all terms with the same powers of $G^{\prime} / G$ and setting each coefficient to zero, we obtain a system of algebraic equations for $a_{0}, a_{1}, v, \lambda$ and $\mu$ as follows:

$\left(\frac{G^{\prime}}{G}\right)^{5}$ coeff.:

$$
-24(c v+\psi) a_{1}+k a_{1}^{5}=0,
$$

$\left(\frac{G^{\prime}}{G}\right)^{4}$ coeff.:

$$
-60(c v+\psi) a_{1} \lambda+5 k a_{0} a_{1}^{4}=0,
$$

$\left(\frac{G^{\prime}}{G}\right)^{3}$ coeff.:

$$
\begin{aligned}
& 2\left(b_{1}-v b_{2}\right) a_{1}+10 k a_{0}^{2} a_{1}^{3}-(c v+\psi)\left(50 a_{1} \lambda^{2}\right. \\
& \left.\quad+40 a_{1} \mu\right)=0,
\end{aligned}
$$

$\left(\frac{G^{\prime}}{G}\right)^{2}$ coeff.:

$$
\begin{aligned}
& 3\left(b_{1}-v b_{2}\right) a_{1} \lambda+10 k a_{0}^{3} a_{1}^{2}-(c v+\psi)\left(60 a_{1} \lambda \mu\right. \\
& \left.\quad+15 a_{1} \lambda^{3}\right)=0, \\
& \left(\frac{G^{\prime}}{G}\right)^{1} \text { coeff.: } \\
& \quad(a-v) a_{1}+\left(b_{1}-v b_{2}\right)\left(2 a_{1} \mu+a_{1} \lambda^{2}\right)+5 k a_{0}^{4} a_{1} \\
& \quad-(c v+\psi)\left(a_{1} \lambda^{4}+22 a_{1} \lambda^{2} \mu+16 a_{1} \mu^{2}\right)=0,
\end{aligned}
$$

$\left(\frac{G^{\prime}}{G}\right)^{0}$ coeff.:

$$
\begin{gathered}
(a-v) a_{0}+\left(b_{1}-v b_{2}\right) a_{1} \lambda \mu+k a_{0}^{5} \\
-(c v+\psi)\left(a_{1} \mu \lambda^{3}+8 a_{1} \lambda \mu^{2}\right)=0 .
\end{gathered}
$$

Solving these under-determined algebraic equations, we obtain the following results:

$$
\begin{aligned}
a & =-\frac{1}{1200 k a_{1}^{4} c^{2}}\left(1728 b_{2}^{2} \psi^{2}-144 b_{2}^{2} \psi k a_{1}^{4}\right. \\
& +3456 b_{1} c b_{2} \psi+1200 c k a_{1}^{4} \psi+1728 b_{1}^{2} c^{2} \\
& \left.-144 b_{1} c b_{2} k a_{1}^{4}-50 c k^{2} a_{1}^{8}+3 b_{2}^{2} k^{2} a_{1}^{5}\right), \\
v & =\frac{k a_{1}^{4}-24 \psi}{24 c}, \\
\mu & =\frac{24\left(b_{1} c+b_{2} \psi\right)+k a_{1}^{4}\left(5 c \lambda^{2}-b_{2}\right)}{20 c k a_{1}^{4}} \\
a_{0} & =\frac{a_{1} \lambda}{2},
\end{aligned}
$$

where $a_{1}, \lambda$ are arbitrary constants.

Substituting the solution set (104) into Eq. (101), the solution formulae of Eq. (100) can be written as

$$
U(z)=a\left\{\frac{\lambda}{2}+\frac{G^{\prime}(z)}{G(z)}\right\} .
$$


Substituting the general solutions of second order linear ODE into Eq. (105) gives two types of traveling wave solutions.

When $\Delta=\lambda^{2}-4 \mu>0$, traveling wave solutions are

$$
\begin{aligned}
& u_{27}(x, t)=\Theta \frac{C_{1} \sinh (\Theta z)+C_{2} \cosh (\Theta z)}{C_{1} \cosh (\Theta z)+C_{2} \sinh (\Theta z)}, \\
& \Theta=\sqrt{\frac{b_{2} k a_{1}^{4}-24\left(b_{1} c+b_{2} \psi\right)}{20 c k a_{1}^{2}}},
\end{aligned}
$$

where $C_{1}$ and $C_{2}$ are arbitrary constants and $z=x-$ $\left\{\frac{k a_{1}^{4}-24 \psi}{24 c}\right\} t$

Next, assuming $C_{1} \neq 0$ and $C_{2}=0$ the traveling wave solution of the perturbed R-KdV-RLW equation can be written as

$$
\begin{aligned}
& u_{28}(x, t)=\Sigma \tanh (\Sigma z), \\
& \Sigma=\sqrt{\frac{b_{2} k a_{1}^{4}-24\left(b_{1} c+b_{2} \psi\right)}{20 c k a_{1}^{4}},}
\end{aligned}
$$

Again, assuming $C_{1}=0$ and $C_{2} \neq 0$, then we obtain $u_{29}(x, t)=\Sigma \operatorname{coth}(\Sigma z)$,

$$
\Sigma=\sqrt{\frac{b_{2} k a_{1}^{4}-24\left(b_{1} c+b_{2} \psi\right)}{20 c k a_{1}^{4}}},
$$

which are shock waves and singular solitary waves, respectively.

When $\Delta=\lambda^{2}-4 \mu<0$,

$$
\begin{aligned}
& u_{30}(x, t)=\Theta \frac{-C_{1} \sin (\Theta z)+C_{2} \cos (\Theta z)}{C_{1} \cos (\Theta z)+C_{2} \sin (\Theta z)}, \\
& \Theta=\sqrt{\frac{b_{2} k a_{1}^{4}-24\left(b_{1} c+b_{2} \psi\right)}{20 c k a_{1}^{4}}},
\end{aligned}
$$

where $C_{1}$ and $C_{2}$ are arbitrary constants and $z=x-$ $\left\{\frac{k a_{1}^{4}-24 \psi}{24 c}\right\} t$

The special assumption $C_{1} \neq 0$ and $C_{2}=0$, leads to the following singular periodic solutions:

$$
\begin{aligned}
& u_{31}(x, t)=-\Sigma \tan (\Sigma z), \\
& \Sigma=\sqrt{\frac{b_{2} k a_{1}^{4}-24\left(b_{1} c+b_{2} \psi\right)}{20 c k a_{1}^{4}},}
\end{aligned}
$$

and when $C_{1}=0, \quad C_{2} \neq 0$ the solution of the perturbed $\mathrm{R}-\mathrm{KdV}-\mathrm{RLW}$ equation will be

$$
\begin{aligned}
& u_{32}(x, t)=\Sigma \tan (\Sigma z), \\
& \Sigma=\sqrt{\frac{b_{2} k a_{1}^{4}-24\left(b_{1} c+b_{2} \psi\right)}{20 c k a_{1}^{4}} .}
\end{aligned}
$$

Remark: The exact solutions (106)-(111) are valid only if

$$
\begin{aligned}
a & =-\frac{1}{1200 k a_{1}^{4} c^{2}}\left(1728 b_{2}^{2} \psi^{2}-144 b_{2}^{2} \psi k a_{1}^{4}\right. \\
& +3456 b_{1} c b_{2} \psi+1200 c k a_{1}^{4} \psi+1728 b_{1}^{2} c^{2} \\
& \left.-144 b_{1} c b_{2} k a_{1}^{4}-50 c k^{2} a_{1}^{8}+3 b_{2}^{2} k^{2} a_{1}^{5}\right) .
\end{aligned}
$$

\section{Exp-function method}

Exp-function method which is proposed by $\mathrm{He}$ and $\mathrm{Wu}$ in 2006 is a straightforward, concise and effective method in obtaining generalized solitary solutions and periodic solutions of nonlinear evolution equations. In comparison with other methods, exp-function method gives more general solutions with some free parameters which, by suitable choice of parameters, result in some known solutions in open literature.

\subsection{Overview of the method}

First, we present briefly the steps of the exp-function method that will be applied.

Consider the general nonlinear partial differential equation of the type

$$
P\left(u, \frac{\partial u}{\partial t}, \frac{\partial u}{\partial x}, \frac{\partial^{2} u}{\partial x^{2}}, \frac{\partial^{2} u}{\partial t \partial x}, \frac{\partial^{2} u}{\partial t^{2}}, \ldots\right)=0 .
$$

Using a transformation

$$
u(x, t)=U(z), \quad z=x-v t,
$$

Eq. (112) can be rewritten as the following nonlinear ODE:

$$
Q\left(U, U^{\prime}, U^{\prime \prime}, \ldots\right)=0 .
$$

According to the exp-function method, we suppose that the wave solution can be expressed in the following form:

$$
U(\xi)=\frac{\sum_{n=-c}^{d} a_{n} \exp (n \xi)}{\sum_{m=-p}^{q} k_{m} \exp (m \xi)},
$$

where $c, d, p$ and $q$ are positive integers which are known to be determined further, $a_{n}$ and $k_{m}$ are unknown constants. We can rewrite Eq. (115) in the following equivalent form:

$$
u(\xi)=\frac{a_{c} \exp (c \xi)+\ldots+a_{-d} \exp (-d \xi)}{k_{p} \exp (p \xi)+\ldots+k_{-q} \exp (-q \xi)} .
$$

This equivalent formulation plays an important and fundamental part in finding the analytic solution of problems. To determine the values of $c$ and $p$, we balance the linear term of highest order of Eq. (114) with the highest order nonlinear term. Similarly, to determine the values of $d$ and $q$, we balance the linear term of lowest order of Eq. (114) with the lowest order nonlinear term. Substituting solution (115) into Eq. (114) yields a set of algebraic equations for $\exp (\xi)$; then all coefficients of $\exp (\xi)$ have to vanish. After this separated algebraic equation, we can find $a_{n}$ and $k_{m}$.

\subsection{Application to $R-K d V-R L W$ equation}

In this subsection, we apply the exp-function method to derive the solution of perturbed $\mathrm{R}-\mathrm{KdV}-\mathrm{RLW}$ equation.

6.2.1. Case $I(n=3)$

Just as before, for $n=3, \mathrm{Eq}$. (66) reduces to

$$
\begin{aligned}
& (a-v) U+\left(b_{1}-v b_{2}\right) U_{z z}-(c v+\psi) U_{z z z z} \\
& \quad+k U^{3}=0 .
\end{aligned}
$$

In order to determine values of $c$ and $p$ in (115), we balance the linear term of highest order in Eq. (117) with the highest order nonlinear term. By simple calculation, 
we have

$$
U^{3}=\frac{c_{1} \exp ((3 c+13 p) z)+\ldots}{c_{2} \exp (16 p z)+\ldots},
$$

and

$$
U^{(4)}=\frac{c_{3} \exp ((c+15 p) z)+\ldots}{c_{4} \exp (16 p z)+\ldots},
$$

where $c_{i}$ are determined coefficients only for simplicity. Balancing highest order of exp-function in Eqs. (118) and (119), we have

$$
3 c+13 p=c+15 p,
$$

which results in

$$
p=c \text {. }
$$

Similarly to determine values of $d$ and $q$, we balance the linear term of lowest order in Eq. (117):

$$
U^{3}=\frac{d_{1} \exp (-(3 d+13 q) z)+\ldots}{d_{2} \exp (-16 q z)+\ldots},
$$

and

$$
U^{(4)}=\frac{d_{3} \exp (-(d+15 q) z)+\ldots}{d_{4} \exp (-16 q z)+\ldots},
$$

where $d_{i}$ are determined coefficients only for simplicity. From Eqs. (122) and (123), we obtain

$$
-(3 d+13 q)=-(d+15 p),
$$

which gives

$$
q=d \text {. }
$$

Choosing $p=c=1$ and $q=d=1$, Eq. (115) becomes

$$
U(z)=\frac{a_{-1} \exp (-z)+a_{0}+a_{1} \exp (z)}{k_{-1} \exp (-z)+k_{0}+k_{1} \exp (z)} .
$$

Substituting Eq. (126) into Eq. (117), and equating to zero the coefficients of all powers of $\exp (n z)$ yield a set of algebraic equations for $a_{0}, k_{0}, a_{1}, a_{-1}, k_{-1}, k_{1}, a$ and $v$. Solving the system of algebraic equations by the help of Maple, we obtain

$$
\begin{aligned}
& a_{0}=a_{0}, \quad k_{0}=k_{0}, \quad k_{1}=k_{1}, \\
& v=\frac{-\psi}{c}, \quad k_{-1}=\frac{k_{0}^{2}\left(b_{1} c+b_{2} \psi\right)+2 k c a_{0}^{2}}{4 k_{1}\left(b_{1} c+b_{2} \psi\right)}, \\
& a=\frac{b_{2} \psi+b_{1} c-2 \psi}{2 c}, \quad a_{1}=\frac{\sqrt{-2 k c\left(b_{1} c+b_{2} \psi\right)} k_{1}}{2 k c}, \\
& a_{-1}=-\frac{\sqrt{-2 k c\left(b_{1} c+b_{2} \psi\right)}\left[k_{0}^{2}\left(b_{1} c+b_{2} \psi\right)+2 k a_{0}^{2} c\right]}{8 k c k_{1}\left(b_{1} c+b_{2} \psi\right)}, \\
& a_{0}=0, \quad a_{1}=a_{1}, \quad k_{-1}=k_{-1}, \quad k_{0}=0, \\
& v=\frac{-\psi}{c}, \quad k_{1}=\frac{\sqrt{-2 k c\left(b_{1} c+b_{2} \psi\right)} a_{1}}{2\left(b_{1} c+b_{2} \psi\right)}, \\
& a=\frac{2\left(b_{1} c+b_{2} \psi\right)-\psi}{c}, \quad a_{-1}=-\frac{2 k_{-1}\left(b_{1} c+b_{2} \psi\right)}{\sqrt{-2 k c\left(b_{1} c+b_{2} \psi\right)}}, \\
& \left.a_{-1}=a-1, \quad k_{0}=k_{0}, \quad 129\right) \\
& v=\frac{-\psi}{c}, \quad a=\frac{b_{1} c+b_{2} \psi-2 \psi}{2 c}, \\
& a_{0}=-\frac{2 k_{0}\left(b_{1} c+b_{2} \psi\right)}{3 \sqrt{-2 k c\left(b_{1} c+b_{2} \psi\right)}}, \quad a_{1}=\frac{5 k_{0}^{2}\left(b_{1} c+b_{2} \psi\right)}{27 k c a_{-1}}, \\
& k_{-1}=\frac{\sqrt{-2 k c\left(b_{1} c+b_{2} \psi\right)} a_{-1}}{b_{1} c+b_{2} \psi},
\end{aligned}
$$

$$
k_{1}=\frac{5 k_{0}^{2}\left(b_{1} c+b_{2} \psi\right)}{36 \sqrt{-2 k c\left(b_{1} c+b_{2} \psi\right)} a_{-1}} .
$$

This in turn gives the following solitary solutions of Eq. (117):

$$
\begin{gathered}
U_{1}(z)=\left[4 k_{1}^{2}\left(b_{1} c+b_{2} \psi\right)\left(\eta k_{1} \mathrm{e}^{z}+2 a_{0} k c\right)-\eta \mathrm{e}^{-z}\left(b_{2} k_{0}^{2} \psi\right.\right. \\
\left.\left.+2 k a_{0}^{2} c+b_{1} c k_{0}^{2}\right)\right] /\left\{2 k c \left[4 k_{1}\left(b_{1} c+b_{2} \psi\right)\left(k_{1} \mathrm{e}^{z}+k_{0}\right)\right.\right. \\
\left.\left.+\left(b_{2} k_{0}^{2} \psi+2 k a_{0}^{2} c+b_{1} k_{0}^{2} c\right) \mathrm{e}^{-z}\right]\right\}, \\
U_{2}(z)=\left[2\left(b_{1} c+b_{2} \psi\right)\left(a_{1} \eta \mathrm{e}^{z}-2 k_{-1} b_{1} c \mathrm{e}^{z}-2 k_{-1} b_{2} \psi \mathrm{e}^{-z}\right)\right] \\
\quad /\left\{\eta\left(\eta a_{1} \mathrm{e}^{z}+2\left(b_{1} c+b_{2} \psi\right) k_{-1} \mathrm{e}^{-z}\right)\right\}, \\
U_{3}(z)=\left\{( b _ { 1 } c + b _ { 2 } \psi ) \left[k_{0}\left(b_{1} c+b_{2} \psi\right)\left(5 k_{0} \eta \mathrm{e}^{z}-48 a_{-1} k c\right)\right.\right. \\
\left.\left.+72 a_{-1}^{2} k c \eta \mathrm{e}^{-z}\right]\right\} /\left\{2 k c \left[k _ { 0 } ( b _ { 1 } c + b _ { 2 } \psi ) \left(5 k_{0}\left(b_{1} c+b_{2} \psi\right) \mathrm{e}^{z}\right.\right.\right. \\
\left.\left.\left.\quad+36 a_{-1} \eta\right)+36 a_{-1}^{2} \eta^{2} \mathrm{e}^{-z}\right]\right\}, \\
\text { where } \\
\eta=\sqrt{-2 k c\left(b_{1} c+b_{2} \psi\right)} .
\end{gathered}
$$

\subsubsection{Case II $(n=5)$}

For $n=5$, Eq. (66) is reduced to

$$
\begin{aligned}
& (a-v) U+\left(b_{1}-v b_{2}\right) U_{z z}-(c v+\psi) U_{z z z z} \\
& \quad+k U^{5}=0 .
\end{aligned}
$$

In order to determine values of $c$ and $p$ in (115), we balance the linear term of highest order in Eq. (132) with the highest order nonlinear term. By simple calculation, we have

$$
U^{5}=\frac{c_{1} \exp ((5 c+11 p) z)+\ldots}{c_{2} \exp (16 p z)+\ldots},
$$

and

$$
U^{(4)}=\frac{c_{3} \exp ((c+15 p) z)+\ldots}{c_{4} \exp (16 p z)+\ldots},
$$

where $c_{i}$ are determined coefficients only for simplicity. Balancing highest order of exp-function in Eqs. (133) and (134), we have

$$
5 c+11 p=c+15 p,
$$

which results in

$$
p=c \text {. }
$$

In a similar way, to determine the values of $d$ and $q$, we balance the linear term of lowest order in Eq. (132):

$$
U^{3}=\frac{d_{1} \exp (-(5 d+11 q) z)+\ldots}{d_{2} \exp (-16 q z)+\ldots},
$$

and

$$
U^{(4)}=\frac{d_{3} \exp (-(d+15 q) z)+\ldots}{d_{4} \exp (-16 q z)+\ldots},
$$

where $d_{i}$ are determined coefficients only for simplicity. From Eqs. (137) and (138), we obtain

$$
-(5 d+11 q)=-(d+15 q),
$$

which gives

$$
q=d \text {. }
$$

Once again, by choosing $p=c=1$ and $q=d=1$, 
Eq. (115) becomes

$$
U(z)=\frac{a_{-1} \exp (-z)+a_{0}+a_{1} \exp (z)}{k_{-1} \exp (-z)+k_{0}+k_{1} \exp (z)} .
$$

Substituting Eq. (141) into Eq. (132), and equating to zero the coefficients of all powers of $\exp (n z)$ yields a set of algebraic equations for $a_{0}, k_{0}, a_{1}, a_{-1}, k_{-1}, k_{1}, a, k$ and $v$. Solving the system of algebraic equations by the help of Maple, we have

$$
\begin{aligned}
& a_{-1}=a_{-1}, \quad a_{0}=a_{0}, \quad k_{-1}=k_{-1}, \quad k_{0}=k_{0}, \\
& v=\frac{b_{1}+5 \psi}{b_{2}-5 c}, \quad a_{1}=\frac{a_{0}^{2} k_{-1}^{2}-a_{-1}^{2} k_{0}^{2}}{4 a_{-1} k_{-1}^{2}}, \\
& a=-\frac{3\left(b_{1} c+b_{2} \psi\right)-2 b_{1}-10 \psi}{2\left(b_{2}-5 c\right)}, \\
& k_{1}=-\frac{a_{0}^{2} k_{-1}^{2}-a_{-1}^{2} k_{0}^{2}}{4 a_{-1}^{2} k_{-1}}, \quad k=\frac{3 k_{-1}^{4}\left(b_{1} c+b_{2} \psi\right)}{2\left(b_{2}-5 c\right) a_{-1}^{4}},
\end{aligned}
$$

which results in the following solution of (132):

$$
\begin{aligned}
& U(z)=\frac{a_{-1}}{k_{-1}} \\
& \times \frac{\left[\left(a_{0}^{2} k_{-1}^{2}+4 a_{-1}^{2} k_{-1}^{2}\right) \mathrm{e}^{-z}+4 a_{0} a_{-1} k_{-1}^{2}-a_{-1}^{2} k_{0}^{2} \mathrm{e}^{z}\right]}{\left[\left(a_{-1}^{2} k_{0}^{2}-a_{0}^{2} k_{-1}^{2}\right) \mathrm{e}^{z}+4 a_{-1}^{2} k_{-1}\left(k_{0}+k_{-1} \mathrm{e}^{-z}\right)\right]} .
\end{aligned}
$$

\section{Conclusions}

This paper studied the dynamics of shallow water waves, modeled by $\mathrm{R}-\mathrm{KdV}-\mathrm{RLW}$ equation comprehensively. There are several solutions that are retrieved from this model, when perturbation terms are taken into account. These are solitary waves, shock waves, singular periodic functions and several more. These solutions come with their respective constraint conditions that must hold for these solutions to exist. Few integration schemes are applied to this paper. The results are overwhelming.

The results of this paper are indeed immensely encouraging to pursue further studies with the model. Later the model will be studied using several numerical schemes. Additionally, stochastic perturbation terms will be taken into account that will lead to the corresponding Langevin equation that will lead to the mean free velocity of the soliton. The results of those research activities are forthcoming.

\section{References}

[1] M. Antonova, A. Biswas, Commun. Nonlin. Sci. Numer. Simulat. 14,734 (2009).

[2] A. Biswas, H. Triki, M. Labidi, Phys. Wave Phenom. 19, 24 (2011).

[3] G. Ebadi, A. Mojavir, H. Triki, A. Yildirim, A. Biswas, Roman. J. Phys. 58, 3 (2013).

[4] A. Esfahani, Commun. Theoret. Phys. 55, 396 (2011)

[5] E.G. Fan, Phys. Lett. A 277, 212 (2000).

[6] M.M. Kabir, A. Khajeh, E. Abdi Aghdam, A. Yousefi Koma, Math. Methods Appl. Sci. 34 213 (2011).
[7] N.A. Kudryashov, J. Appl. Math. Mech. 52, 361 (1988).

[8] N.A. Kudryashov, Commun. Nonlin. Sci. Numer. Simulat. 17, 2248 (2012).

[9] N.A. Kudryashov, Phys. Lett. A 147, 287 (1990).

[10] N.A. Kudryashov, Phys. Lett. A 155, 269 (1991).

[11] M. Labidi, A. Biswas, Math. Eng. Sci. Aerospace 2, 183 (2011).

[12] W.X. Ma, Phys. Lett. A 180, 221 (1993).

[13] W.X. Ma, B. Fuchssteiner, Int. J. Nonlin. Mech. 31, 329 (1996).

[14] W. Malfliet, Phys. Scr. 54, 563 (1996).

[15] W. Malfliet, Phys. Scr. 54, 569 (1996).

[16] P. Razborova, H. Triki, A. Biswas, Ocean Eng. 63, 1 (2013).

[17] P. Razborova, B. Ahmed, A. Biswas, Appl. Math. Inf. Sci. 8, 485 (2014).

[18] P. Razborova, L. Moraru, A. Biswas, Roman. J. Phys. 59, 658 (2014).

[19] P. Razborova, A.H. Kara, A. Biswas, Nonlin. Dyn. 79, 743 (2015).

[20] P.N. Ryabov, Appl. Math. Comput. 217, 3585 (2010).

[21] P. Sanchez, to appear in Roman. J. Phys..

[22] M.L. Wang, X.Z. Li, J.L. Zhang, Phys. Lett. A 372, 417 (2008).

[23] A.M. Wazwaz, Appl. Math. Comput. 159, 559 (2004).

[24] A.M. Wazwaz, Math. Comp. Model. 40, 499 (2004)

[25] A.M. Wazwaz, Appl. Math. Comput. 154, 713 (2004)

[26] E. Zayed, K.A. Gepreel, Appl. Math. Comput. 212, 1 (2009).

[27] S. Zhang, J.L. Tong, W. Wang, Phys. Lett. A 372, 2254 (2008).

[28] H. Zhang, Commun. Nonlin. Sci. Numer. Simulat. 14, 3220 (2009).

[29] J.-M. Zuo, Appl. Math. Comput. 215, 835 (2009).

[30] J.-M. Zuo, Y.-M. Zhang, T.-D. Zhang, F. Chang, Boundary Value Problems 2010, 516260 (2010). 